\title{
FATORES ASSOCIADOS AO DESEMPENHO ESCOLAR: RESULTADOS DE UM MODELO MULTINÍVEL DE VALOR ADICIONADO
}

PAULO A. MEYER M. NASCIMENTO

\section{RESUMO}

Este artigo estima um modelo multinível de valor agregado para uma amostra de escolas, turmas e alunos matriculados na $2^{\underline{a}}$ série do ensino fundamental em Salvador (BA), em uma tentativa de identificar fatores associados a desempenho escolar. Ademais, o presente estudo destaca as complicações metodológicas que decorrem da omissão de variáveis relevantes, passivel de ocorrer quando recursos são endogenamente determinados - e descreve métodos de tratar esse problema. Ainda que os resultados obtidos não permitam inferências sobre potenciais ligações causais entre as variáveis explicativas e a variável dependente, eles sugerem que, ao menos para a amostra estudada, desempenho em testes padronizados de Leitura estaria associado apenas ao conhecimento prévio do estudante, à escolaridade de seus pais e ao tipo de escola (pública ou privada) em que estudava na ocasião da aplicação dos instrumentos de coleta de dados do primeiro ano de campo do Projeto GERES.

PALAVRAS-CHAVE: RENDIMENTO ESCOLAR • FUNÇÃO SOCIAL - QUALIDADE DO ENSINO - PROJETO GERES. 


\section{RESUMEN}

Este artículo considera un modelo multi nivel de valor agregado para una muestra de escuelas, grupos y alumnos inscriptos en el segundo grado de la enseñanza fundamental en Salvador (BA), en un intento de identificar factores asociados al desempeño escolar. Además, el presente estudio destaca las complicaciones metodológicas provenientes de omisiones de variables relevantes, hecho que puede ocurrir cuando se determinan recursos de foma endógena. También se describen métodos para tratar el problema. Aunque los resultados obtenidos no permitan inferir sobre potenciales relaciones causales entre las variables explicativas y la variable dependiente, sugieren que, al menos para la muestra estudiada, el desempeño en pruebas estandarizadas de lectura estaría asociado apenas al conocimiento previo de los alumnos, a la escolaridad de sus padres y al tipo de escuela (pública o privada) en la que estudiaba cuando se aplicaron los instrumentos de recolección de datos del primer año de campo del Proyecto GERES.

PALABRAS CLAVE: RENDIMIENTO ESCOLAR • FUNCIÓN SOCIAL • CALIDAD DE LA ENSEÑANZA・PROJETO GERES

\section{ABSTRACT}

This paper analyzes a multilevel model of value added in a sample of schools, classes and students enrolled in the second grade of elementary school in Salvador, state of Bahia, in an attempt to identify factors associated with school performance. In addition, this study highlights the methodological complications arising from the omission of relevant variables, which may occur when resources are endogenously determined, and describes methods of dealing with this problem. Although the results do not allow inferences about potential causal links between explanatory variables and the dependent variable, they suggest that, at least for the sample examined, performance in standardized reading tests is associated only with students' prior knowledge, their parents' education and the type (public or private) of school they were attending when the data collecting instruments were applied during the first year of the GERES Project field work.

KEYWORDS: LEARNING ACHIVIEMENT • SOCIAL FUNCTION • EDUCATION QUALITY • GERES PROJECT. 


\section{FATORES ASSOCIADOS AO DESEMPENHO ESCOLAR}

$O$ aproveitamento de um estudante em cada etapa e ao longo de seu ciclo escolar é resultado de uma série de fatores concorrentes que muitas vezes escapam dos limites da escola e, em decorrência disso, políticas educacionais implementadas isoladamente e/ou em descompasso com um conjunto de outras políticas públicas (Saúde, Saneamento, Emprego e Renda, Assistência Social, etc.) tendem a apresentar resultados insatisfatórios (IPEA, 2010). Em meio a tamanha confluência de fatores (escolares, pessoais, familiares, sociais e institucionais), aqueles que têm sido de maior consenso na Academia como determinantes de desempenho escolar são os que remetem ao nível socioeconômico dos educandos. Assim tem sido mundo afora desde a publicação do Relatório Coleman (Coleman, 1966).

Elaborado para o governo norte-americano com o intuito de investigar desigualdades educacionais, esse relatório concluiu que as condições familiares e sociais seriam os principais determinantes de desempenho escolar. Permanece ele hoje tal qual uma espécie de "pedra fundamental", tanto na literatura de Economia da Educação, cuja evidência empírica assenta-se, sobretudo, 
em estimativas de parâmetros de funções de produção da educação quanto na literatura de eficácia escolar, que se propõe a ir além de uma relação "insumo-produto" e abrir a "caixa-preta" do processo educativo que resulta em sucesso (ou fracasso) escolar (LeVAČić, 2005; NASCIMENTO, 2008).

Sem ir de encontro ao consenso geral de que as desigualdades socioeconômicas dão o contorno das desigualdades educacionais, as pesquisas pós-Relatório Coleman têm avançado, sob os mais diferentes ângulos, na busca por fatores relacionados à escola positivamente associados a melhores desempenhos estudantis e que, portanto, ensejem a adoção de políticas educacionais mais capazes de minimizar os efeitos das desigualdades socioeconômicas sobre o aprendizado e o percurso escolar do educando.

No âmbito da pesquisa de eficácia escolar, por exemplo, um bom desempenho escolar é observado quando o estudante consegue progredir além do esperado dadas as suas características pessoais, seu conhecimento prévio e seu capital social, cultural e econômico. Nesta ótica, eficaz é a escola que agrega mais valor ao resultado escolar de seus alunos comparativamente a outras cujo contexto em que estão inseridas lhe é similar-mostrando-se essa interferência positiva no desempenho acadêmico associada a políticas e práticas escolares ali implementadas (CASTRO, 2009, citando Mortimore, 1991 e SOARes; TORres; Alves, 2002).

Já em Economia da Educação, as perguntas de investigação mais recorrentes costumam ser de duas naturezas: i) se há variáveis (e quais) ao nível da escola, particularmente relacionadas a recursos e à sua aplicação, que apresentam algum impacto sobre o desempenho, ou ao menos a ele estejam sistematicamente associadas em um dado contexto; ii) qual intervenção, dentre políticas alternativas a serem consideradas, incorre em menor custo de oportunidade, ou, em outros termos, tende a obter maior relação benefício/custo uma vez adotada.

No Brasil, tal qual no restante da América Latina, a pesquisa educacional que utilize métodos quantitativos mostra-se historicamente escassa (Morduchowicz, 2004), com pouca receptividade nas faculdades de educação (ВrоoкE, 2005) e de reduzida apropriação pelos formuladores de política (IOSCHPE, 2004). Não obstante, em anos recentes tem surgido no país um crescente número de estudos que utilizam métodos quantitativos 
para identificar determinantes de desempenho e eficácia escolar (WAiselfisZ, 2000; BARros et AL., 2001; AlbernaZ, Ferreira; Franco, 2002; De Felicio; Fernandes, 2005; Curi; MenezesFilho, 2006; Menezes-Filho; Pazello, 2007; Menezes-Filho, 2007; Biondi; De Felício, 2007; Alves; SoARes, 2008; Machado Et Al., 2008; CASTRO, 2009; De OliveirA, 2010).

O presente trabalho aproxima-se mais do campo explorado por economistas da educação na busca de fatores associados ao desempenho escolar. Sem adentrar na dinâmica da sala de aula e do aprendizado, objeto de estudo mais próprio da literatura sobre eficácia escolar, estima uma função de produção da educação na qual uma medida de desempenho (nota em um exame padronizado) é regressado por proxies de um conjunto de fatores escolares, pessoais, familiares e institucionais comumente destacados pela literatura como determinantes do desempenho.

Para tanto, estimar-se-á um modelo de valor agregado que, por permitir efeitos randômicos, avança em relação às funções de produção da educação que não levam em conta a natureza de clusters própria dos dados educacionais. Representa ainda um diferencial a presença de uma medida inicial de desempenho como variável de controle, por permitir considerar que um dado desempenho acadêmico decorra não somente das condições pessoais, escolares, familiares, sociais e institucionais às quais esteja submetido o educando no momento do exame, mas também de um histórico de experiências de vida que lhe tenham ajudado a construir um conjunto de percepções, de habilidades e de conhecimentos prévios que o auxiliam naquele momento e ao longo de sua vida.

Conforme se argumentará adiante, o estudo ora apresentado, ao dispor de uma medida inicial de desempenho, consegue reduzir os efeitos de potenciais vieses de seleção às características não-observadas que porventura estejam sistematicamente associadas ao desenvolvimento de capacidades, habilidades e conhecimentos do indivíduo, ou que tenham sido alteradas entre a medida inicial e as demais medidas de desempenho disponíveis. Por isso, e diante da impossibilidade de se obter um instrumento ou um estimador de matching adequado o suficiente para extirpar a contento o problema decorrente da endogeneidade da proxy atinente aos recursos escolares, não há que se falar doravante em "impactos" 
ou "efeitos" das variáveis explicativas sobre a variável dependente do modelo; o que ora se investiga, outrossim, é o quão associadas podem estar aquelas à medida final de desempenho estudantil (o $Y$ da regressão) na amostra estudada de 4.025 estudantes e 169 turmas de segunda série do Ensino Fundamental das 55 escolas urbanas, públicas e privadas, de Salvador (BA) que integram a amostra do Projeto GERES ${ }^{1}$.

A próxima seção discorre sobre os problemas metodológicos que emergem quando viés de seleção tende a estar presente na amostra. A seção seguinte apresenta a base de dados e o método utilizados. Na sequência são apresentados os resultados do modelo. A seção final traz as considerações finais.

\section{VIÉS DE SELEÇÃO E SUAS IMPLICAÇÕES}

Durante as três décadas que se seguiram à publicação do supracitado Relatório Coleman, proliferavam-se estimativas de funções de produção da educação que pareciam ignorar que esta não poderia ser construída de maneira completamente análoga à função de produção de uma firma. Afinal, em um processo educativo não se pode supor que seus insumos (professores, infraestrutura física da escola, materiais de ensino) relacionados ao resultado de interesse (notas em testes padronizados, por exemplo) sejam exógenos ao modelo.

Particularmente no que tange à relação entre o tamanho das turmas (proxy aqui utilizada para recursos escolares) e o desempenho em testes padronizados, o problema da endogeneidade ou viés de seleção decorre do fato de estudantes não serem, na maioria dos casos, alocados em suas turmas de maneira aleatória. A composição e o tamanho das classes costumam ser resultado, em geral, das interações entre as decisões e os comportamentos dos agentes envolvidos no processo educativo, isto é, dos pais, dos docentes, dos gestores e dos próprios alunos, sintetizadas tal como segue:

- pais com maior envolvimento na educação de seus filhos e que valorizem turmas menores podem matriculá-los em escolas que ofereçam uma média inferior de estudantes por classe, ou pressionar gestores (diretores ou dirigentes) a reduzir o tamanho das turmas (de suas escolas ou sistemas de ensino);

1 Transcrevendo definição extraída do próprio site do Projeto, "o GERES é um projeto de pesquisa longitudinal que focaliza a aprendizagem nas primeiras fases do Ensino Fundamental para estudar os fatores escolares e sociofamiliares que incidem sobre o desempenho escolar" (extraído de www.geres.ufmg.br. Acesso em: 05 de maio de 2011). Ver Franco, Brooke e Alves (2008) para uma apresentação do desenho metodológico do estudo longitudinal em que consiste o Projeto GERES e do qual o presente trabalho utiliza parte dos dados relacionados ao primeiro de seus quatro anos de duração. 
- gestores escolares podem ter políticas próprias, explicitadas formalmente ou não, de alocação de estudantes a diferentes turmas;

- docentes podem adotar comportamentos e estratégias diferentes a depender do tamanho e da composição da turma em que lecionam;

- alguns sistemas públicos de ensino podem ter regras próprias de formação de turmas, ensejando, por exemplo, políticas compensatórias que aloquem estudantes com maior dificuldade de aprendizagem em turmas menores e/ou com professores mais capacitados - ou mesmo o contrário, isto é, políticas que privilegiem os alunos de melhor desempenho, colocando-os nas menores classes e/ou com os melhores docentes.

Portanto, quando a formação das turmas está sujeita a tais influências é de esperar que existam variáveis nãoobservadas passíveis de afetar o número de alunos por sala. Em assim sendo, como seriam endógenas ao modelo, essas variáveis não-observadas estariam, por conseguinte, correlacionadas aos termos de erro. Com isto, independentemente do tamanho da amostra, estimativas obtidas a partir de minimos quadrados ordinários deixam de representar o melhor estimador não enviesado e consistente do parâmetro populacional (VIGNoLEs ET AL., 2000; WEBBINK, 2005; WOOLDRIDGE, 2002).

\subsection{MÉTODOS PARA TRATAR A ENDOGENEIDADE}

O problema da endogeneidade ou viés de seleção costuma ser enfrentado por um conjunto de métodos econométricos alternativos. Os principais deles estão resumidamente descritos a seguir.

\subsubsection{EXPERIMENTOS RANDÔMICOS CONTROLADOS}

A melhor maneira de verificar possíveis efeitos causais, coeterisparibus, é rodar um experimento aleatório com um grupo de tratamento e outro de controle. Isto porque, quando bem feito, experimentos assim eliminam eventuais efeitos de diferenças pré-existentes entre indivíduos que possam afetar resultados finais. De fato, alocando-se aleatoriamente 
indivíduos a um grupo de tratamento e a outro de controle garante que as variáveis investigadas não estejam relacionadas (e, portanto, tampouco correlacionadas) a diferenças no desempenho entre os dois grupos (Glewwe, 2002; Averett; MClennan, 2004).

Entretanto, experimentos em educação são custosos e costumam enfrentar dilemas éticos e barreiras políticas nada desprezíveis, além de serem passíveis de sofrer vieses decorrentes de perdas na amostra ao longo do experimento (atrito) e difíceis de replicar em populações mais amplas (Levačić, 2005). Por essa razão, a aplicabilidade de seus resultados é limitada a intervenções específicas, na realidade onde o próprio experimento ocorreu (Todd; Wolpin, 2003). Ademais, ainda quando experimentos são possíveis e discentes e docentes são alocados aleatoriamente a turmas de diferentes tamanhos, é impossível garantir que aqueles que participam do experimento não modifiquem seu comportamento por saberem ser parte de um (HOXBY, 2000; AVERETT; MCLENNAN, 2004).

Em face dessas questões, muitos pesquisadores costumam contestar a validade dos resultados de experimentos como o American Student/TeacherAchievementRatio (STAR), mais famoso dos experimentos com tamanho de turmas relatados na literatura e cujos resultados apontavam, em geral, melhor desempenho em turmas menores (KRUEGER, 2003). No Brasil, não há registros difundidos de experiências dessa natureza.

\subsubsection{VARIÁVEIS INSTRUMENTAIS}

Pesquisadores usam variáveis instrumentais para obter estimadores consistentes quando variáveis relevantes tendem a estar omitidas do modelo (WoOLDRIDGE, 2002). Isto pode ocorrer quer porque não há dados disponíveis para medi-las, quer porque a teoria em que se baseia o pesquisador não tenha ainda identificado precisamente como reconhecê-las ou mesmo medi-las. Dispondo de uma variável instrumental apropriada, o pesquisador torna-se capaz de isolar a parte exógena da variação explicativa que deseja incluir no modelo, pois uma e outra se correlacionam, mas o instrumento não afeta a variável dependente diretamente.

Variáveis instrumentais têm sido bastante utilizadas em diversos países para lidar com a endogeneidade 
de variáveis concernentes à qualidade do ensino (Steele; VignOles; Jenkins, 2007). Costuma-se derivá-las basicamente de regrasinstitucionaisou variações naturais(NASCIMENTO, 2008; WebBink, 2005).

Para o Brasil, Menezes-Filho e Pazello (2007) utilizam a reforma que introduziu o Fundef como instrumento em um modelo que relaciona a proficiência dos estudantes com o salário dos professores, mantidas constantes as características dos estudantes, dos docentes e das escolas. A justificativa para usar o Fundef como instrumento reside no fato de ter sido instituído por uma reforma conduzida a nível federal, mas que repercutia no financiamento das escolas estaduais e municipais. Vale destacar, porém, que as mudanças introduzidas por regras institucionais como o Fundef podem circunstancialmente gerar variações exógenas no eventual efeito dos salários dos professores sobre o desempenho estudantil - mas tal desdobramento tende a durar apenas o tempo necessário para que os agentes as assimilem e incorporem seus efeitos às suas tomadas de decisão, o que limitaria a validade do resultado às observações do período imediatamente após a adoção das novas regras, e apenas àquelas referentes às escolas para as quais a regra de fato afetou diretamente (Нохву, 200o). Ademais, a adoção de variáveis instrumentais costuma vir acompanhada de críticas acerca do quão defensáveis seriam elas do ponto de vista teórico. Afinal, este método parte da premissa de que não são as variáveis instrumentais correlacionadas com a variável dependente - e sustentar tal premissa exige substancial embasamento (MoRgAn; WINSHIP, 2007).

Há na literatura dois exemplos clássicos de variáveis instrumentais utilizadas para contornar especificamente a endogeneidade em modelos cuja proxy para recursos escolares é o tamanho das turmas. Angrist e Lavy (1999) usam uma regra vigente em Israel que impõe um limite máximo de alunos por turma, enquanto Hoxby (2000) utiliza variações naturais nas taxas de natalidade de diferentes coortes de estudantes. Como não há, no presente trabalho, nem dados comparáveis referentes a diferentes coortes, nem a ocorrência de um fator não-esperável que cause variações exógenas no tamanho das turmas, variáveis instrumentais não foram utilizadas. 


\subsubsection{MATCHING}

Este método busca isolar o efeito de uma política (tal qual redução do tamanho das turmas) ao comparar dois conjuntos de unidades de análise que se mostram idênticos em todas as características observáveis relevantes, exceto que um desses conjuntos recebe um 'tratamento' (classes menores) e o outro, não. A forma mais difundida de obter esse 'tratamento' é por meio de um escore $b(X)$ que exprime a probabilidade de uma dada unidade de análise integrar um grupo de tratamento, dadas as características $X$ observadas, de tal forma que a distribuição condicional de $X$ em $b(X)$ seja independente da alocação de cada unidade isolada ao grupo de tratamento ou ao grupo de controle - ocasião em que $b(X)$ é conhecido como propensity score matching (CALIENDo; KoPEINIG, 2008).

A principal dificuldade com este método é sua sensibilidade à escolha dos condicionantes $X$ - em verdade, para utilizar estimadores de matching é necessário assentar-se sobre uma base teórica bastante sólida e dispor de dados apropriados e em quantidade para que seja fortemente defensável a distribuição das unidades de análise entre os grupos de tratamento e de controle pelo critério estabelecido e de acordo com os condicionantes adotados (HeckMan; NAVARro-LozANo, 2004).

No Brasil, De Oliveira (2010) aplica este método aos dados do Sistema Nacional de Avaliação da Educação Básica (Saeb) de 2005 para identificar o impacto de políticas de redução do tamanho da classe e de aumento da jornada escolar. A autora chega à conclusão que, confrontando-se essas duas políticas a partir dos dados do Saeb de 2005, a ampliação da jornada escolar de quatro para cinco horas apresenta maior razão benefício-custo quando as classes não ultrapassam o número de 33 estudantes, sendo mais custo-efetiva a política de redução das turmas quando estas têm maior quantidade de estudantes. Para o presente trabalho, contudo, a obtenção de um estimador de matching não se mostrou viável, tendo em vista que tal procedimento reduziria de tal maneira o tamanho da amostra que o contrafactual ao "experimento da redução do tamanho das turmas" não seria representativo nem mesmo para o conjunto de estudantes que compôs a amostra. 


\subsubsection{MODELOS DE FRONTEIRA}

Trata-se de modelos que se valem de técnicas tanto paramétricas (fronteiras estocásticas) quanto não-paramétricas (análise envoltória de dados) para tentar identificar, no caso de estudos educacionais, a eficiência de escolas individuais em relação à fronteira da função de produção pertinente (VIGNOles et Al., 200o). Isto significa que tais métodos permitem identificar, dentro de um conjunto de escolas, aquelas que obtêm o melhor desempenho de seus estudantes, dados os recursos disponíveis.

No Brasil, modelos de fronteira aplicados a dados educacionais costumavam ser escassos até o início dos anos 2000. Nos últimos anos, alguns estudos que o fazem têm surgido (particularmente utilizando análises envoltórias de dados). Especificamente em relação à Educação Básica, ver Gasparini e Ramos (2003), Delgado e Machado (2007), Delgado (2009) e Sampaio e Guimarães (2009). Para a proposta do presente artigo, contudo, esses modelos não são tão pertinentes, tendo em vista que concernem a fatores exógenos capazes de afetar a eficiência produtiva das escolas - e não a variações nos recursos disponíveis que possam estar associados ao aproveitamento do aluno.

\subsubsection{MODELOS DE VALOR ADICIONADO}

Modelos de valor adicionado (ou agregado) incluem na função de produção uma medida anterior de desempenho do estudante, seja como uma variável de controle, seja como um componente da variável dependente (por exemplo, Y sendo definida como a diferença entre a nota obtida no momento $\mathrm{t}_{1}$ e a nota obtida no momento $\mathrm{t}_{0}$ ). Este procedimento aprimora modelagens de funções de produção da educação com uma única equação, uma vez que potencialmente controla, até o momento $t_{0}$, o histórico do contexto escolar e familiar capaz de influenciar o desempenho em $t_{1}$.

A mensuração correta do valor adicionado exige uma medida inicial de conhecimento prévio e uma ou mais medidas do aproveitamento do aluno ao longo do ciclo escolar objeto de estudo. Com isso, é possível medir o progresso do estudante ao longo do tempo. Os dados do Projeto GERES permitem isso por dispor de uma nota decorrente de uma avaliação aplicada 
no momento $\mathrm{t}_{0}$, ou seja, antes de transcorrido o ciclo escolar que é objeto de estudo.

Esse tipo de informação não costuma ser ainda disponível no Brasil. Albernaz, Ferreira e Franco (2002) mencionam a base de dados do Saresp como uma possível fonte de informações para especificar modelos de valor agregado no Brasil; esse estudo, porém, sugere ser suficiente, para essa finalidade, que haja duas medidas de desempenho de um mesmo aluno em diferentes pontos do tempo - embora dessa forma, conforme argumentado no presente trabalho, seja negligenciado o controle para o conhecimento prévio desenvolvido pelo aluno até o momento $t_{0}$ da pesquisa, o que exigiria uma medida inicial de desempenho obtida a priori.

Alguns estudos tentam obter alguma medida de valor agregado a partir de métodos que, a rigor, não o fazem, por não acompanhar o progresso acadêmico do indivíduo. ${ }^{2}$ Assim o fazem, por exemplo, Franco e Menezes-Filho (2008), que utilizam a diferença no desempenho médio das escolas no Saeb entre $t_{1}$ e $t_{0}$, e Luz (2006) que, valendo-se de um estudo feito pelo Inep entre 2000 e 2001 com o mesmo conjunto de estudantes, utiliza para tal finalidade as notas obtidas em testes padronizados de Matemática aplicados ao final da $5^{\mathrm{a}}$ e da $6^{\mathrm{a}}$ séries - e que, portanto, medem construtos diferentes e aferem apenas desempenhos em etapas diversas do ciclo escolar, não necessariamente progressos no aprendizado.

Modelos de valor adicionado, ainda quando corretamente especificados, não são suficientes, contudo, para superar completamente o problema da endogeneidade, tendo em vista ser possível que efeitos dos recursos educacionais à disposição do estudante estejam sistematicamente relacionados às características familiares (que podem inclusive variar entre $t_{0}$ e $t_{1}$ ) e a desempenhos anteriores até mesmo a $\mathrm{t}_{0}$.

É por isso que, mesmo que as estimativas dos parâmetros para tamanho das turmas mostrem-se significativas no presente trabalho, não há que se falar em relações de causa e efeito desta com desempenho. Isto porque, sem um tratamento estatístico adequado para a endogeneidade, tais tendências que porventura fossem verificadas poderiam estar a sinalizar comportamentos e decisões dos agentes, e não eventuais efeitos de reduções do tamanho das turmas 
nas notas dos alunos. Para tornar essa questão mais clara, basta ter em mente que algumas escolas podem preferir formar turmas menores com alunos de maior dificuldade de rendimento (situação na qual a função de produção estimada poderá vir a indicar que turmas menores pioram o desempenho), enquanto em outras o padrão poderá ser de turmas menores para os melhores estudantes (caso em que a função de produção tenderá a indicar desempenho melhor quanto menor as turmas).

Como não foi possível obter nenhuma variável instrumental solidamente defensável do ponto de vista teórico, nem tampouco um estimador de matching pertinente, não se buscou mensurar eventuais efeitos de quaisquer das variáveis explicativas sobre a variável dependente do modelo. O que foi investigado, vale ressaltar uma vez mais, foi se haveria alguma associação das variáveis construídas (e que buscavam mensurar fatores usualmente apontados na literatura como relevantes ao desempenho escolar) às notas em testes padronizados de Leitura no contexto estudado.

\section{BASE DE DADOS E MÉTODO APLICADO}

Os dados ora utilizados referem-se a um conjunto de 4.025 estudantes matriculados, em 2005, na segunda série do Ensino Fundamental de 55 escolas e 169 turmas das redes estadual, municipal e privada de Salvador (BA). Não se trata, contudo, da amostra inicial do Projeto GERES, que intentava ser representativa do universo de alunos das escolas urbanas públicas e privadas da capital baiana que cursavam a segunda série do ensino fundamental em 2005 - ver Andrade e Miranda (2004) para maior detalhamento do procedimento de amostragem do Projeto GERES. O conjunto de observações utilizados no presente trabalho é menor que a amostra inicial por esta já ter sido afetada pelo atrito do primeiro ano. Para torná-lo representativo do universo ao qual se propunha inicialmente investigar, ao conjunto de observações aqui utilizado precisariam ser aplicados pesos amostrais. Entretanto, estes ainda não estavam disponíveis no momento de elaboração do presente trabalho. 
Assim, não é possível estender os resultados da pesquisa ora apresentada ao universo de estudantes, turmas e escolas urbanas de segunda série do ensino fundamental de Salvador, nem mesmo ao conjunto de escolas GERES da capital baiana. Os resultados doravante reportados são válidos apenas para o conjunto de estudantes presentes nas duas primeiras ondas do Projeto GERES. Isto não os invalida, contudo, como exercício de testagem da associação entre tamanho das turmas e desempenho escolar.

Foram aplicados testes padronizados de Leitura em dois momentos do ano acadêmico de 2005 (março e novembro). A aplicação de um teste no início do ano permitiu a aferição de uma medida inicial de desempenho a ser usada como proxy para conhecimento prévio dos estudantes. Com isso, foi possível construir um modelo de valor agregado para o conjunto de estudantes da amostra.

Por meio dos questionários aplicados em novembro daquele ano às famílias e aos professores dos alunos, foi possível incluir nesse modelo proxies para fatores escolares e familiares costumeiramente apontados pela literatura como potencialmente associados ao desempenho nas provas: as características do professor e do lar da criança. Os dados disponíveis permitiram, ainda, a inclusão no modelo de um fator institucional já identificado como relevante no contexto brasileiro ${ }^{3}$ : se a escola é pública ou privada.

Por fim, os registros administrativos de cada escola, também disponíveis, permitiram obter o número de estudantes alocados em cada turma da amostra. Assim, o modelo inclui o logaritmo natural do número de alunos por turma como proxy para recursos escolares, outro fator normalmente incluído em funções de produção da educação, embora a maioria dos estudos empíricos não encontre nele significância como determinante de desempenho escolar ${ }^{4}$. A opção pela forma logarítmica encontra respaldo em Hoxby (2000), que salienta ser não-linear a relação esperada entre tamanho da turma e desempenho - isto é, espera-se que o eventual impacto de se reduzir em uma a quantidade de crianças alocadas em uma turma seja maior quando nessa turma estudavam inicialmente 25 do que quando em $\mathrm{t}_{0}$ eram 40 as crianças ali matriculadas.

3 Ver, por exemplo, Menezes-Filho (2007), que, utilizando dados do Saeb 2003, encontra, mesmo controlando para diferenças socioeconômicas das famílias, escolaridade dos pais e características escolares, diferenças no desempenho médio das escolas públicas e privadas da ordem de $18 \%$ o que atribui possivelmente a diferenças de gestão.

4 Para uma revisão de literatura sobre a relação entre recursos e desempenho, vide Nascimento (2008). Para evidência empírica especificamente sobre o tamanho das turmas e desempenho no contexto brasileiro, vide Waiselfisz (2000), que utiliza dados do Saeb de 1997 para a região Nordeste, e De Oliveira (2010) cujo estudo parte de dados do Saeb de 2005 
5 Ver em Jaeger e Holm (2007) justificativa, baseada

na teoria da reprodução social de Bordieu, para a utilização da escolaridade dos pais como proxy para capital cultural das famílias. Ver, ainda, em Checchi (2006) que a escolaridade dos pais não deve ser incluída em uma função de produção da educação como determinante do desempenho, uma vez que tende ela a ser multicolinear à renda e ao comportamento dos pais, bem como a habilidades inatas da criança e a outras características não-observáveis que possam influenciar o desempenho escolar. Por isso, essa variável é aqui incluída como controle para capital cultural da família, não como variável explicativa do modelo.
Dessa forma, chegou-se a um modelo de valor agregado que é dado por:

$$
Y_{i j k 1}=\propto+\theta Y_{i j k o}+\beta Z_{j k}+\varsigma T_{j k}+w F_{i j k}+\varphi I+v_{k}+\mu_{j k}+\epsilon_{i j k}
$$

Onde:

- $Y_{i j k 1}$ mede o desempenho, na prova de Leitura aplicada ao final do ano acadêmico de 2005, do(a) estudante $i$, matriculado na turma $j$ da escola $k$.

- $Y_{i j k 0}$ mede o conhecimento prévio, dado pela nota obtida em Leitura no início (março) do ano acadêmico de 2005 pelo(a) estudante $i$ da classe $j$ da escola $k$.

- $Z_{j k}$ é o logaritmo natural (log) do tamanho da turma $j$ da escola $k$.

- $T_{j k}$ é um vetor que compreende duas características ligadas ao docente da turma $j$ na escola $k$ : sua escolaridade (medida por uma dummy que assume o valor de 1 quando o professor possui nível superior) e sua experiência (fator construído a partir de análise dos componentes principais das respostas dadas a três perguntas constantes do questionário do professor e que se referem ao tempo em que ensina, ao tempo em que ensina na escola $k$ e ao tempo em que leciona naquela série ou ciclo específicos).

- $F_{i j k}$ é um vetor para insumos familiares, compreendendo duas variáveis. A primeira é a escolaridade dos pais, tomada aqui, quando disponível para ambos os genitores, a do que apresentar maior escolaridade, com o intuito de representar, com ela, um conjunto de fatores relevantes ao capital cultural presente na família, manifestados em características a ela associadas, porém, não diretamente observáveis, a exemplo dos gostos, preferências e percepções gerais da família em relação à educação do filho e ao sistema educacional que ele freqüenta ${ }^{5}$. A outra variável consiste em um fator calculado por análise de componentes principais que visa captar o capital econômico das famílias: ativos da família mede os ativos físicos a que tem acesso a criança em casa, tais como quantidade de televisores, computadores, refrigeradores, telefones etc.

- I é um vetor para os termos de interação entre $Z$ e $T$. 
- $v_{k}, \cup_{j k}$ e $\epsilon_{i j k}$ são os resíduos respectivamente para escolas, turmas e estudantes.

Este modelo leva em consideração que os dados educacionais têm natureza hierárquica. Estudantes são alocados a turmas, as quais, por sua vez, compõem uma determinada escola e um dado sistema escolar - portanto, indivíduos de uma mesma turma estão submetidos a condições e experiências mais semelhantes entre si do que em relação a seus pares de outras turmas, assim como os de uma mesma escola tendem a ter mais em comum entre si do que os de escolas diferentes e os de um mesmo sistema de escola idem, e assim por diante.

Essa estrutura hierárquica dos dados pode acarretar superestimação de parâmetros, caso a modelagem aplicada a ignore. No limite, a não aplicação de modelos hierárquicos (também chamados de multiníveis) a análises de dados educacionais dessa natureza pode levar a inferências imprecisas que incorretamente levem o pesquisador a rejeitar a hipótese nula (SNiJders; BoSKER, 1999; FerRão, 2006). Muitos economistas costumam tentar fugir desse problema por meio de uma estratégia que consiste em: a) agregar os dados (normalmente ao nível da turma, mas às vezes até ao nível da escola ou mesmo do sistema de ensino); b) incluir algumas variáveis (em geral, dummies) no nível da escola e/ou do sistema de ensino com o intuito de incorporar esses efeitos. Esta estratégia é amplamente utilizada, por exemplo, por WößmanneWest (2006). Agregação, entretanto, também tende a induzir vieses (HANUsheK; Rivkin; TAYLOR, 1996). Além disso, impedem conclusões acerca dos indivíduos, uma vez que qualquer tentativa nesse sentido com base apenas em dados no nível de grupos seria contaminada pela falácia ecológica - interpretação errônea dos dados que atribui a indivíduos parâmetros estatísticos que se referem tão somente aos grupos de que eles fazem parte (GOldSTEIN, 1995; SNIJDERS; BosKer, 1999).

Evidencia-se o quão em clusters estão os dados da amostra pesquisada a partir dos componentes da variância em cada nível (aluno, turma e escola) - cujas estimativas para as variáveis $Y_{1}$ e $Z$ estão informadas na tabela 1 a seguir.

31 Est. Aval. Educ., São Paulo, v. 23, n. 51, p. 16-39, jan./abr. 2012 
* Todas as estimativas são para modelos multiniveis especificados separadamente para $Y_{i}$ e $Z$, utilizando-se IGLS (sigla em inglês para iterativos mínimos quadrados generalizados).

TABELA 1 - Componentes da variância e correlações

intra-níveis para $Y_{1}$ e $Z^{*}$

\begin{tabular}{|c|c|c|}
\hline & ESTIMATIVA & ERRO PADRÃO \\
\hline \multicolumn{3}{|c|}{$\begin{array}{l}Y_{1} \\
\text { (desempenho no teste de Leitura } \\
\text { aplicado em novembro de 2005) }\end{array}$} \\
\hline Entre escolas & 0,883 & 0,245 \\
\hline $\begin{array}{l}\text { Entre turmas de uma } \\
\text { mesma escola }\end{array}$ & 0,667 & 0,116 \\
\hline $\begin{array}{l}\text { Entre estudantes de uma } \\
\text { mesma turma }\end{array}$ & 4,877 & 0,111 \\
\hline Correlação intraescola & 0,141 & - \\
\hline Correlação intraturma & 0,104 & - \\
\hline \multicolumn{3}{|l|}{$\begin{array}{l}Z \\
\text { (log do tamanho da turma) }\end{array}$} \\
\hline Entre escolas & 0,040 & 0,008 \\
\hline $\begin{array}{l}\text { Entre turmas de uma } \\
\text { mesma escola }\end{array}$ & 0,049 & 0,001 \\
\hline Correlação intraescola & 0,449 & - \\
\hline
\end{tabular}

Fonte: Cálculos próprios feitos no MLwiN 2.0 com dados do Projeto GERES

De posse de tais estimativas, é possível calcular as correlações intraturma e intraescola por:

$$
\rho(\mathbf{x})=\frac{\sigma_{x}^{2}}{\sigma_{i}^{2}+\sigma_{j}^{2}+\sigma_{k}^{2}}
$$

Onde $x$ representa cada escola, nas ocasiões em que o efeito da escola está sendo calculado, ou cada turma, quando a fórmula está a calcular o efeito da turma; $\rho$ é o efeito em questão (chamado de coeficiente de correlação intra-cluster) e $\sigma_{i}^{2} \sigma_{i}^{2}$ e $\sigma_{k}^{2}$ são as três partes da variância total, decomposta, respectivamente, nos componentes referentes ao aluno, à turma e à escola.

Conforme destacado em Steele, Vignoles e Jenkins (2007), correlações dessa natureza podem ser interpretadas, em um modelo de intercepto randômico, como a correlação entre as notas esperadas de dois estudantes escolhidos ao acaso dentro de uma mesma turma de uma dada escola. As estimativas da tabela 1 mostram que $24,5 \%$ do total da variância nas notas de Leitura devem-se às diferenças entre turmas e entre escolas, ao passo que $44,9 \%$ da variância total do tamanho das turmas ocorrem entre escolas. Isto indica que aproximadamente um quarto da variabilidade das notas é explicada não pela diferença entre as crianças 
em si, mas pela heterogeneidade das turmas e das escolas da amostra; e que quase metade da variabilidade do tamanho das turmas ocorre dentro das próprias escolas, e não entre elas.

Como resultado, a aplicação de um modelo hierárquicolinear em três níveis (aluno, turma e escola), tal qual proposto, torna-se mais adequado, uma vez que modelos que tratem como fixos esses efeitos nos níveis macro tendem a incorrer em vieses de agregação e, mais grave, a superestimar os coeficientes das variáveis cuja variância organiza-se em clusters. Modelagens multiníveis tendem a reduzir, portanto, os riscos de se cometer erros do tipo I (rejeitar a hipótese nula quando, em verdade, ela permanece válida).

\section{RESULTADOS}

O modelo de valor adicionado especificado na seção anterior foi estimado no pacoteestatístico MLwiN2.0, por meio doalgoritmo iterativos mínimos quadrados generalizados (IGLS, na sigla em inglês - sobre esse algoritmo, vide GoLDSTEIN, 1986), implementado nesse software especializado em modelagens multiníveis. Os resultados obtidos estão apresentados na tabela 2 abaixo.

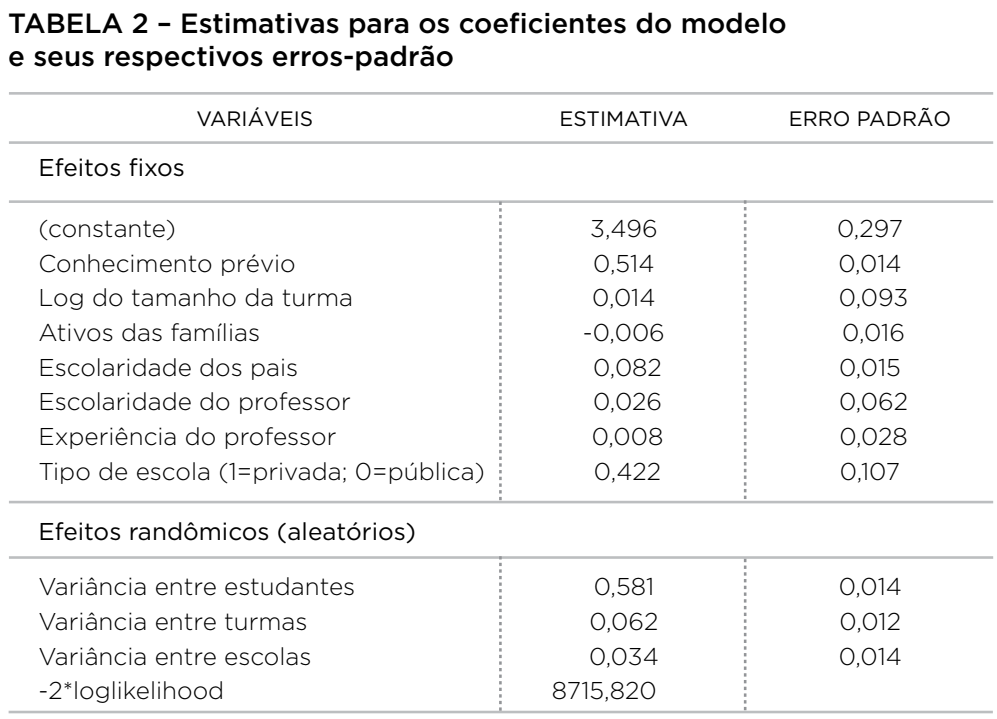

Fonte: Cálculos próprios feitos no MLwiN 2.0 com dados do Projeto GERES. 
Os resultados apresentados sugerem que, ao menos para o conjunto de estudantes avaliados, o que mais interferiu no aproveitamento escolar ao final daquele ano foram:

- a própria bagagem de conhecimentos que a criança trazia de anos anteriores;

- o capital cultural de sua família (medida pela escolaridade dos pais);

- e o tipo de escola (pública ou privada) na qual frequentou a série em questão.

Isto porque somente as variáveis conhecimento prévio, escolaridade dos pais e tipo de escola mostraram-se significativas a 95\% de intervalo de confiança. A variável tamanho das turmas, proxy para recursos escolares, não se mostrou significante, como tampouco o foram as variáveis concernentes a características do professor e ao capital econômico das famílias. Esse retrato prevaleceu, inclusive, com a adição ao modelo de termos de interação entre a variável tamanho das turmas e as demais variáveis atinentes à sala de aula (especificamente, a escolaridade e a experiência docente). Quando a variável dependente passou a ser a diferença entre as notas do fim e do início do ano (ou seja, quando o progresso do aluno foi a variável de interesse), a variável tipo de escola deixou de ser significante, sinalizando que as escolas privadas da amostra não fizeram melhor do que as públicas no que tange ao progresso acadêmico de seus alunos.

É possível que a variável tamanho da turma não tenha sido significante por não haver na amostra turmas muito grandes. De fato, a média de alunos por turma foi de 25,5, abaixo dos 33 que Oliveira (2010) identifica como limite para que políticas de redução de turmas apresentem benefícios insuficientes para justificar seus custos - para classes com menos de 33 alunos, Oliveira (2010) estima como mais custo-efetiva uma política de ampliação da jornada escolar do que de redução do número de alunos por classe. Mesmo as maiores não passaram de 36 estudantes. De todo modo, sem uma variável instrumental ou um estimador de matching apropriados para superar a contento os vieses de seleção próprios de modelos desta natureza, não é possível fazer inferências confiáveis a respeito da influência ou não desta variável no desempenho das crianças da amostra usada no presente trabalho. 
Quanto às variáveis concernentes aos atributos do professor, é possível que não sejam as proxies utilizadas as mais apropriadas para medi-los. Embora sejam as mais utilizadas, até por serem mais comumente disponíveis, a escolaridade e a experiência do docente não costuma apresentar coeficientes significativos em estudos que as utilizam. Segundo Ioschpe (2004), o domínio demonstrado pelo professor da matéria que leciona é que tem sido mais apontado pela literatura como importante para o desempenho escolar. No entanto, dados que meçam tal domínio ainda são poucos no Brasil e não estavam disponíveis na base do Projeto GERES.

\section{CONSIDERAÇÕES FINAIS}

Em fase final de compilação e tratamento estatístico, a base GERES permitirá avançar no estudo ora apresentado, mediante a elaboração de modelos multiníveis longitudinais que superem muitas das limitações metodológicas aqui incorridas. Ainda assim, os dados seccionais utilizados permitiram a construção de um modelo de valor agregado mais apropriado do que os que fazem uso de fontes de dados educacionais que não contêm medidas iniciais de desempenho para um mesmo aluno, como a Prova Brasil e o Saeb, por exemplo.

Ademais, lançou luz sobre o grau de associação com o desempenho estudantil que apresentam, para o conjunto de estudantes avaliados nas duas primeiras ondas do Projeto GERES em Salvador, alguns dos fatores pessoais, familiares, escolares e institucionais tidos na literatura como mais relevantes para $o$ aprendizado.

\section{REFERÊNCIAS BIBLIOGRÁFICAS}

ANGRIST, J. D.; LAVY, V. Using Maimonides' rule to estimate the effect of class size on scholastic achievement. The Quarterly Journal of Economics, v. 114, n. 2, p. 533, 1999.

ALBERNAZ, A.; FERREIRA, F. H. G.; FRANCO, C. Qualidadee e equidade no ensino fundamental brasileiro. Pesquisa e Planejamento Econômico, v. 32 , n. 3, p. $453-476$, dez. 2002. 
ALVES, M. T. G.; SOARES, J. F. O Efeito das escolas no aprendizado dos alunos: um estudo com dados longitudinais no Ensino Fundamental. Educação e Pesquisa, v. 34, n. 3, 2008.

ANDRADE, D. F.; MIRANDA, E. C. M. GERES: relatório técnico do plano amostral (Relatório Amostral). Belo Horizonte: Projeto GERES, 2004. Disponível em: <www.geres.ufmg.br>. Acesso em: 1 set. 2007.

AVERETT, S. L.; McLENNAN, M. C. Exploring the effect of class size on student achievement: what have we learned over the past two decades? In: G. Johnese J. Johnes (Eds.), International Handbook on the Economics of Education. Cheltenham: Elgar, 2004.

BARROS, R. P.; MENDONÇA, R.; SANTOS, D. D.; QUINTAES, G.

Determinantes do desempenho educacional no Brasil. Texto para Discussão. n. 834. Rio de Janeiro: Ipea, 2001.

BIONDI, R. L.; DE FELíCIO, F. Atributos escolares e o desempenho dos estudantes: uma análise em painel dos dados do Saeb. Brasília: Inep, 2007.

BLATCHFORD, P. The Class size debate: is small better? Maidenhead: UK Open UP, 2003.

BLATCHFORD, P. et al. A study of class size effects in English School Reception Year classes. British Educational Research Journal, 28, p. 169-185, 2002.

BROOKE, N. The GERES Project: a longitudinal study of the 2005 school generation in Brazil. Revista Eletrônica Ibero-americana Sobre Qualidade, Eficácia e Mudança em Educação, v. 3, 2005.

CALIENDO, M.; KOPEINIG, S. Some practical guidance for the implementation of propensity score matching. Journal of Economic Surveys, v.22, n. 1, p. 31-72, 2008.

CASTRO, R. F. Fatores associados ao desempenho escolar na $4^{\mathrm{a}}$ série do ensino fundamental. In: LORDÊLO, J. A.; DAZZANI, M. V. (Org.). Avaliação educacional: desatando e reatando nós. Salvador: EDUFBA, 2009, p. 265-296.

CHECCHI, D. The Economics of Education: human capital, family background and inequality. Cambridge: Cambridge UP, 2006.

COLEMAN, J. S. Equality of educational opportunity. Washington, D.C.: US Office of Education, 1966.

CURI, A. Z.; MENEZES-FILHO, N. A Relação entre o desempenho escolar e os salários no Brasil. Texto para Discussão, n. 3/2006. São Paulo: IBMEC, 2006.

DE FELÍCIO, F.; FERNANDES, R. O Efeito da Qualidade da Escola Sobre o Desempenho Escolar: uma avaliação do ensino fundamental no Estado de São Paulo. Encontro Nacional de Economia, 33. Natal, Anais do... 2005.

DELGADO, V. M. S.; MACHADO, A. F. Eficiência das escolas públicas estaduais de Minas Gerais. Pesquisa e Planejamento Econômico, v. 37, n. 3, dez. 2007.

DELGADO, V. M. S. Estudo sobre um ranking municipal de eficiência escolar em Minas Gerais. Revista Brasileira de Estudos Regionais e Urbanos, v. 2, n. 1, 2009. 
DE OLIVEIRA, J. M. Custo-efetividade de políticas de redução do tamanho da classe e de ampliação da jornada escolar: uma aplicação de estimadores de matching. Revista do BNDES, n. 33, p. 281-305, jun. 2010.

FRANCO, C.; BROOKE, N.; ALVES, F. Estudo longitudinal sobre qualidade e equidade no ensino fundamental: GERES 2005. Ensaio: Avaliação e Políticas Públicas em Educação, v. 16, n. 61, p. 625-638, 2008.

FRANCO, A. M. P.; MENEZES-FILHO, N. Uma análise de rankings de escolas brasileiras com dados do Saeb. Encontro Nacional de Economia, 36. Salvador: Anais do..., 2008.

FERRÃO, M. E. Componentes do efeito-escola no Brasil. In: Francisco Javier Murillo Torrecilla (Org). Estudios sobre eficacia escolar en Iberoamérica: 15 buenas investigaciones. Bogotá: Convenio Andrés Bello, 2006.

GASPARINI, E. C.; RAMOS, F. S. Efetividade e eficiência no ensino médio brasileiro. Economia Aplicada, v. 7, n. 2, p. 389-411, abr./jun.2003.

GLEWWE, P. Schools and skills in developing countries: education policies and socioeconomic outcomes. Journal of Economic Literature, n. 40 , p. 436-482, 2002.

GOLDSTEIN, H. Multilevel mixed linear model analysis using iterative generalizes least squares. Biometrika, v. 73, n. 1, p. 43-56, abr. 1986.

Multilevel Statistical Models. 2. ed. Londres: Edward Arnold, 1995.

HANUSHEK, E. A.; RIVKIN, S.; TAYLOR, L. Aggregation and the estimated effects of school resources. The Review of Economics and Statistics, n. 78, p. 611-627, 1996.

HECKMAN, J. J.; NAVARRO-LOZANO, S. Using matching, instrumental variables, and control functions to estimate economic choice models. The Review of Economics and Statistics, n. 86, p. 30-57, 2004.

HOXBY, C. The effects of class size on student achievement: new evidence from population variation. The Quarterly Journal of Economics, n. 115 , p. $1.239-1.285,2000$.

IBGE - INSTITUTO BRASILEIRO DE GEOGRAFIA E ESTATÍSTICA. Censo Demográfico 2000. Rio de Janeiro: IBGE, 2003.

IOSCHPE, G. A Ignorância custa um mundo: o valor da educação no desenvolvimento do Brasil. São Paulo: Francis, 2004.

IPEA - INSTITUTO DE PESQUISA ECONÔMICA APLICADA. Perspectivas para a promoção da educação como direito de todos. In: Perspectivas da política social no Brasil. Brasília: IPEA, 2010.

JÆGER, M. M.; HOLM, A. Does parents' economic, cultural, and social capital explain the social class effect on educational attainment in the Scandinavian mobility regime? Social Science Research, n. 36, p. 719-744, 2007.

KRUEGER, A. B. Economic considerations and class size. The Economic Journal, n.113, p. F34-F63, 2003.

37 Est. Aval. Educ., São Paulo, v. 23, n. 51, p. 16-39, jan./abr. 2012 
LEVAČIĆ, R. The resourcing puzzle: the difficulties of establishing causal links between resourcing and student outcomes. London: The Institute of Education, University of London, 2005.

LUZ, L. S. Os Determinantes do desempenho escolar: a estratificação educacional e o efeito valor adicionado. Encontro Nacional de Estudos Populacionais, 15. Caxambu: Anais do..., 2006.

MACHADO, A. F. et al. Qualidade do ensino em Matemática: determinantes do desempenho de alunos em escolas públicas estaduais mineiras. Revista Economia, v. 9, n. 1, p. 23-45, jan.-abr. 2008.

MENEZES-FILHO, N. A. Determinantes do desempenho escolar do Brasil. São Paulo: Instituto Futuro Brasil, IBMEC-SP e USP, 2007.

MENEZES-FILHO, N.; PAZELLO, E. Do teachers' wages matter for proficiency? Evidence from a funding reform in Brazil. Economics of Education Review, v. 26, n. 6, p. 660-672, 2007.

MORDUCHOWICZ, A. Discusiones de Economia de la Educacion. Buenos Aires: Losada, 2004.

MORGAN, S. L.; WINSHIP, C. Counterfactuals and causal inference: methods and principles for social research. Nova Iorque (EUA): Cambridge University Press, 2007.

NASCIMENTO, P. A. M. M. School resources and student achievement: Worldwide findings and methodological issues. Educate $\sim$ Special Issue, p. 19-30, mar. 2008.

OLIVEIRA, J. M. Custo-efetividade de políticas de redução do tamanho da classe e de ampliação da jornada escolar: uma aplicação de estimadores de matching. Revista do BNDES, n. 33, p. 281-305, jun. 2010.

SAMPAIO, B.; GUIMARÃES, J. Diferenças de eficiência entre ensino público e privado no Brasil. Economia Aplicada, v. 13, n. 1, p. 45-68, jan.-mar. 2009.

SNIJDERS, T. A. B.; BOSKER, R. J. Multilevel Analysis: an introduction to basic and advanced multilevel modeling. Londres: Sage Publications, 1999.

STEELE, F.; VIGNOLES, A.; JENKINS, A. The effect of school resources on pupil attainment: a multilevel simultaneous equation modeling approach. Journal of Royal Statistical Society-Series A,170, 2007.

TODD, P. E.; WOLPIN, K. I. On the specification and estimation of the production function for cognitive achievement. Economic Journal, n .113, p. F3-F33, 2003.

VIGNOLES, A.; et al. The Relationship between Resource Allocation and Pupil Attainment: a review. Nottingham: DfEE, 2000.

WAISELFISZ, J. J. Tamanho da turma: faz diferença? Brasília: FUNDESCOLA/MEC, 2000 (Série Estudos, n. 12), 2000.

WEBBINK, D. Causal effects in education. Journal of Economic Surveys, n. 19, p. 535-560, 2005. 
WOOLDRIDGE, J. M. Introductory Econometrics: a modern approach.

2.ed. Nova Iorque: South-Western, 2002.

WÖßMANN, L. ;West, M. Class-size effects in school systems around the World: evidence from between-grade variation in TIMSS.

European Journal of Political Economy, n. 50, p. 695-736, 2006.

PAULO A. MEYER M. NASCIMENTO

Técnico de Planejamento e Pesquisa da Diretoria

de Estudos e Políticas Setoriais, de Inovação,

Regulação e Infraestrutura (Diset) do Ipea

paulo.nascimento@ipea.gov.br

Recebido em: DEZEMBRO 2011

Aprovado para publicação em: JANEIRO 2012 